\title{
Effect of non-abrupt doping and interfacial profiles on the carrier sheet density in one-side modulation-doped GaN/AIGaN quantum wells
}

\author{
B. G. Enders, F. M. S. Lima, A. L. A. Fonseca, and O. A. C. Nunes \\ Instituto de Física, Universidade de Brasília, Cx. Postal 04455, \\ 70919-970, Brasília, DF, Brazil \\ E. F. da Silva Jr. \\ Departamento de Física, \\ Universidade Federal de Pernambuco, Cidade Universitária \\ 50670-901, Recife, PE, Brazil \\ (Received on 1 September, 2008)
}

\begin{abstract}
The results of an accurate theoretical study on the effects of non-abrupt doping and interfacial profiles on the electron sheet density in one-side modulation-doped wurtzite GaN/AlGaN single quantum wells at low temperatures are presented. We solve coupled Schrödinger and Poisson equations self-consistently via the finite difference method. By employing a proper discretization on a nonuniform grid and taking into account the strong piezoelectric and spontaneous polarization fields exhibited by the wurtzite III-nitride heterostructures, a substantial increase in the 2DEG density is predicted with the increase of the donor diffusion length and the reduction of the spacer thickness.
\end{abstract}

Keywords: non-abrupt doping; interfacial profiles; carrier sheet density; quantum wells

\section{INTRODUCTION}

The effects of non-abrupt doping and interfacial profiles on the performance of a variety of electronic devices have been already identified $[1,2]$. It was found, for example, that high-electron mobility transistors (HEMTs) have their threshold voltage and the rate of gate-voltage switching strongly dependent on the composition and the doping profile along the growth direction [3], which also affects the performance of one-side modulation-doped quantum well (QW) infrared photodetectors [4]. Theoretically, better device performances should be observed with the use of heterostructures with abrupt doping profiles, a fact that made the reduction of impurity interdiffusion an important research topic in semiconductor physics. However, there are experimental evidences indicating that the actual doping profiles are not abrupt $[5,6]$ and this is due to limitations in the control of impurity implantation during the growth process, as well as to dopant diffusion/segregation during the deposition and posterior annealing $[7,8]$. Additionally, there is also experimental evidence supporting the existence of graded interfaces in GaN/AlGaN quantum wells $[9,10]$.

In this work, we present the results of an accurate theoretical study on the effects of non-abrupt doping and interfacial profiles on the electron sheet density in a graded oneside modulation-doped GaN/AlGaN QW at low temperature. The electronic subband structure is determined by solving the coupled Schrödinger and Poisson differential equations selfconsistently via finite difference method (FDM), employing a proper discretization on a nonuniform grid [11], and taking into account the strong piezoelectric and spontaneous polarization fields exhibited by the wurtzite III-nitride heterostructures [12]. Emphasis is given on the dependence of the carriers sheet density on both the spacer thickness and the donors diffusion length.

\section{GOVERNING EQUATIONS}

The electron eigenstates are calculated self-consistently by solving the one-electron Schrödinger equation within the effective mass and Hartree approximations [13] along with the Poisson equation for the quantum well under consideration at a given temperature and for a given graded distribution of donor impurities. A linear variation of the alloy molar fraction $x$ into the heterointerfaces is considered (see Fig. 1). As there are differences in both the carrier effective mass and the dielectric constant along the growth direction (chosen here as the $z$-axis), the BenDaniel-Duke Hamiltonian [14] for the Schrödinger equation must be employed, yielding

$$
-\frac{\hbar^{2}}{2} \frac{\mathrm{d}}{\mathrm{d} z}\left[\frac{1}{m^{*}(z)} \frac{\mathrm{d} \psi_{j}(z)}{\mathrm{d} z}\right]+V(z) \psi_{j}(z)=E_{j} \psi_{j}(z),
$$

where $\hbar$ is the reduced Plank constant, $m^{*}(z)$ is the positiondependent effective mass, $\psi_{j}(z)$ is the envelope wavefunction for the $j$-th subband, $E_{j}$ is the corresponding energy eigenvalue, and $V(z)=V_{0}(z)+V_{H}(z)$ is the potential energy, $V_{0}(z)$ being the potential energy before the charge transfer process and $V_{H}(z)$ being the Hartree potential.

The Hartree potential is the solution of the Poisson equation. As the dielectric constant varies along the $z$-axis, one has to make use of the generalized Poisson equation [15], which reads

$$
\frac{\mathrm{d}}{\mathrm{d} z} \varepsilon(z) \frac{\mathrm{d}}{\mathrm{d} z} V_{H}(z)=\frac{e^{2}}{\varepsilon_{0}}\left[N_{D}^{+}(z)-n(z)\right],
$$

where $\varepsilon(z)$ is the position-dependent dielectric constant, $e$ is the elementary charge, and $\varepsilon_{0}$ is the vacuum permittivity constant. The charge concentrations on the right-hand side of Eq.(2) are $N_{D}^{+}(z)$, for ionized donors, and $n(z)=$ $\sum_{j=1}^{J} N_{s}^{(j)}\left|\psi_{j}(z)\right|^{2}$, for electrons, $j$ being the subband index, $N_{S}^{(j)}$ being the carrier sheet density at the $j$-th subband, and $J$ identifies the highest occupied subband.

The spontaneous and piezoelectric polarizations in wurtzite GaN-based quantum wells are taken into account here in the 


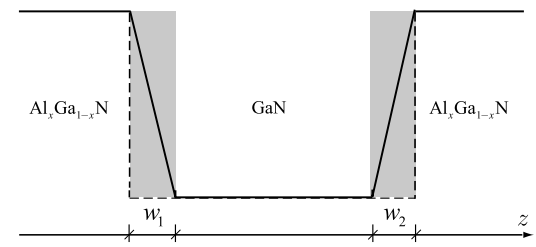

FIG. 1: The linear interfacial profile adopted for the alloy molar fraction variation in a single graded GaN/AlGaN QW with asymmetric interfaces of thicknesses $w_{1}$ and $w_{2}$.

form of polarization fields, which are included in the bare potential as follows:

$$
V_{0}(z)= \begin{cases}e F_{b} z+\Delta E_{c}, & \text { for } z<0 \\ e F_{w} z, & \text { for } 0<z<L_{w} \\ e\left(F_{b} z+F_{w} L_{w}\right)+\Delta E_{c} & \text { for } z>L_{w}\end{cases}
$$

where $L_{w}\left(L_{b}\right)$ is the well (barrier) width, $F_{w}\left(F_{b}\right)$ is the magnitude of the electric field in the well (barrier), and $\Delta E_{c}$ is the $\mathrm{GaN}-\mathrm{AlGaN}$ conduction-band offset, assumed to be given by $\Delta E_{c}=0.75\left[E_{g}(x)-E_{g}(0)\right][16,17]$, where [18]

$$
E_{g}(x)=6.13 x+3.42(1-x)-1.0 x(1-x)
$$

is the $\mathrm{Al}_{x} \mathrm{Ga}_{1-x} \mathrm{~N}$ band gap in $\mathrm{eV}$.

By considering that the overall potential drop across the structure has to be null, i.e. $F_{w} L_{w}+F_{b} L_{b}=0$, then the polarization fields can be written in terms of the total polarization in the well, $P_{w}$, and in the barrier, $P_{b}$, as [19]:

$$
F_{w, b}=\frac{\left(P_{b, w}-P_{w, b}\right) L_{b, w}}{\varepsilon_{w, b} L_{b, w}+\varepsilon_{b, w} L_{w, b}},
$$

in which $\varepsilon_{w}\left(\varepsilon_{b}\right)$ is the dielectric constant of the well (barrier). The total polarizations can be calculated directly by the sum of the piezoelectric $\left(P^{\mathrm{pz}}\right)$ and spontaneous $\left(P^{\mathrm{sp}}\right)$ polarizations of the corresponding layers:

$$
P_{w, b}=P_{w, b}^{\mathrm{sp}}+P_{w, b}^{\mathrm{pz}} .
$$

For $\mathrm{Al}_{x} \mathrm{Ga}_{1-x} \mathrm{~N} / \mathrm{GaN} \mathrm{QW}$ grown on $\mathrm{GaN}$ buffer we have $P_{w}^{\mathrm{pz}}=0$ and $P_{w}^{\mathrm{sp}}=-0.034 \mathrm{C} / \mathrm{m}^{2}$, and the remaining polarization expressions for the strained alloy can be written as (in $\left.\mathrm{C} / \mathrm{m}^{2}\right)[18]$

$$
\begin{gathered}
P_{b}^{\mathrm{sp}}(x)=-0.090 x-0.034(1-x)+0.021 x(1-x), \\
P_{b}^{\mathrm{pz}}(x)=2 \frac{a(x)-a_{0}}{a_{0}}\left[e_{31}(x)-e_{33}(x) \frac{C_{13}(x)}{C_{33}(x)}\right],
\end{gathered}
$$

where $a(x)$ is the basal lattice constant of the wurtzite IIInitride alloy, $a_{0}$ is the lattice constant of the $\mathrm{GaN}$ buffer, $e_{31}, e_{33}$ are the piezoelectric constants and $C_{13}, C_{33}$ are the elastic constants [20]. These parameters are predicted to follow the composition-weighted average between the relevant binary compound values, i.e. Vegard's rule. These values are presented in Tab. I.

In the depletion approximation, it is considered that all impurities are ionized within a layer of width $L_{D}$ [23]. In this
TABLE I: Wurtzite nitride binaries parameters considered in the composition-weighted average used in the determination of the ternary nitride alloys parameters.

\begin{tabular}{cccc}
\hline \hline Parameters & GaN & AlN & InN \\
\hline$a(\AA)^{a}$ & 3.189 & 3.112 & 3.545 \\
$e_{31}\left({\left.\mathrm{C} / \mathrm{m}^{2}\right)^{b}}^{b}\right.$ & -0.34 & -0.53 & -0.41 \\
$e_{33}\left(\mathrm{C} / \mathrm{m}^{2}\right)^{b}$ & 0.67 & 1.50 & 0.81 \\
$C_{13}(\mathrm{GPa})^{a}$ & 106 & 108 & 92 \\
$C_{33}(\mathrm{GPa})^{a}$ & 398 & 373 & 224 \\
$m^{* a}$ & $0.22 m_{0}$ & $0.30 m_{0}$ & $0.14 m_{0}$ \\
\hline \hline
\end{tabular}

${ }^{a}$ Ref. 21.

${ }^{b}$ Ref. 22.

work we have surpassed this approximation by considering a more realistic position-dependent concentration of ionized impurities as given by [24]

$$
N_{D}^{+}(z)=\frac{N_{D}(z)}{1+2 \exp \left[\frac{E_{F}+E_{D}-V(z)}{k_{B} T}\right]},
$$

in which $E_{D}$ in the donor ionization energy, $E_{F}$ is the Fermi level, $T$ is the temperature, and $N_{D}(z)$ is the total concentration of impurities, described here by means of a normalized error function, $\operatorname{erf}(x)$, via the following expression:

$$
N_{D}(z)=\frac{N_{D}^{0}}{2}\left[1-\operatorname{erf}\left(\frac{z+L_{s}}{2 D_{l}}\right)\right],
$$

where $D_{l}$ is the donor diffusion length, $N_{D}^{0}$ is the nominal doping concentration, and $L_{s}$ is the spacer thickness.

Finally, the 2DEG areal density in each subband is obtained by means of

$$
N_{s}^{(j)}=\frac{m^{*} k_{B} T}{\pi \hbar^{2}} \ln \left[1+\exp \left(\frac{E_{F}-E_{j}}{k_{B} T}\right)\right] .
$$

Having established the governing equations, we can now apply the FDM for solving Eqs. (1) and (2) for a GaN/AlGaN QW in a self-consistent manner. In doing this, it was shown that stable and accurate numerical results are obtained only if the so-called proper discretization is adopted [14]. We verified that such discretization may be generalized for nonuniform meshes in order to achieve an even higher level of precision and stability [11]. For the Schrödinger equation, the following difference equation was adopted:

$$
-\frac{\hbar^{2}}{2}\left[a_{i} \psi_{i-1}^{(j)}-\left(a_{i}+b_{i}\right) \psi_{i}^{(j)}+b_{i} \psi_{i+1}^{(j)}\right]+V_{i} \psi_{i}^{(j)}=E_{j} \psi_{i}^{(j)},
$$

where the index $i$ identifies the grid points along the $z$-axis direction. The auxiliary parameters $a_{i}$ and $b_{i}$ are given by

$$
\begin{aligned}
a_{i} & \equiv\left(m_{i-1 / 2}^{*} \Delta z_{i} \Delta z_{i+1 / 2}\right)^{-1} \\
b_{i} & \equiv\left(m_{i+1 / 2}^{*} \Delta z_{i+1} \Delta z_{i+1 / 2}\right)^{-1},
\end{aligned}
$$

with

$$
\begin{aligned}
m_{i \pm 1 / 2}^{*} & =\frac{m_{i}^{*}+m_{i \pm 1}^{*}}{2} \\
\Delta z_{i+1 / 2} & =\frac{\Delta z_{i}+\Delta z_{i+1}}{2}
\end{aligned}
$$




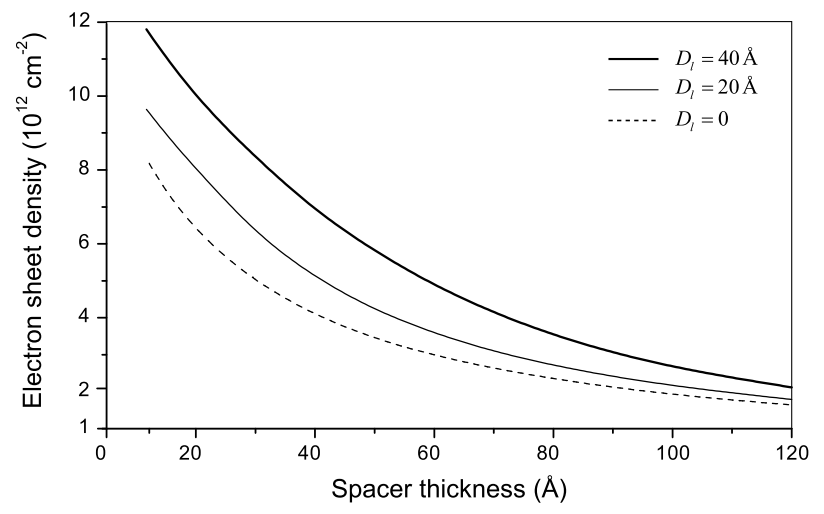

FIG. 2: Dependence of electron sheet density on spacer thickness in a $60 \AA$ wide graded $\mathrm{GaN} / \mathrm{Al}_{0.8} \mathrm{Ga}_{0.2} \mathrm{~N}$ QW with a normal interface width of $w_{1}=3 \mathrm{ML}$ and an inverted interface width of $w_{2}=4 \mathrm{ML}$. The nominal donor concentration is $N_{D}^{0}=6 \times 10^{18} \mathrm{~cm}^{-3}$.

where $\Delta z_{i}$ represents the $i$-th mesh step size. By using the same discretization procedure for the generalized Poisson equation, one has

$$
\begin{array}{r}
\frac{\varepsilon_{i-1 / 2}}{\Delta z_{i}} V_{H_{i-1}}-\left(\frac{\varepsilon_{i-1 / 2}}{\Delta z_{i}}+\frac{\varepsilon_{i+1 / 2}}{\Delta z_{i+1}}\right) V_{H_{i}}+\frac{\varepsilon_{i+1 / 2}}{\Delta z_{i+1}} V_{H_{i+1}} \\
=\frac{e^{2}}{\varepsilon_{0}} \Delta z_{i+1 / 2}\left(N_{D_{i}}^{+}-n_{i}\right)
\end{array}
$$

where

$$
\varepsilon_{i \pm 1 / 2}=\frac{\varepsilon_{i}+\varepsilon_{i \pm 1}}{2}
$$

The charge transfer iterations are simulated by successive changes in the quasi-Fermi levels in both the well and the barrier layers, until the difference between them is reduced to less than a small chosen tolerance, when the thermodynamic equilibrium is reached.

\section{RESULTS AND DISCUSSION}

In Fig. 2, the dependence of the carriers sheet density $\left(=\sum_{j=1}^{J} N_{s}^{(j)}\right)$ on $L_{s}$ is shown for a $60 \AA$-wide wurtzite graded $\mathrm{GaN} / \mathrm{Al}_{0.2} \mathrm{Ga}_{0.8} \mathrm{~N} \mathrm{QW}$ with a normal interface width of $w_{1}=$ $3 \mathrm{ML}$ and an inverted interface width of $w_{2}=4 \mathrm{ML}$ (see Fig. 1), one monolayer (ML) for GaN being $2.59 \AA$ long. The dashed line corresponds to the abrupt doping profile in the depletion approximation while solid lines are related to the non-abrupt doping profiles with donors diffusion length of $D_{l}=20 \AA$ (thin line) and $D_{l}=40 \AA$ (thick line). A nominal donor concentration of $N_{D}^{0}=6 \times 10^{18} \mathrm{~cm}^{-3}$ was considered.

As seen in Fig. 2, for all values of $D_{l}$ the 2DEG density decreases rapidly for $L_{s}$ below, say, $60 \AA$. Note also that the 2DEG density increases significantly with the increase of $D_{l}$ from 0 (abrupt) to $40 \AA$. The increase of $L_{s}$ tends to bring the curves closer due to the reduction of the effect of the nonabruptness of the doping profile on the charge transfer process.

Hence, a significant increase in $N_{S}$ (up to $64 \%$ for $L_{S} \approx 30 \AA$ ) is predicted when non-abrupt doping profiles are taken into account. Though this demands more elaborate computational programming, the more realistic results obtained with our non-abrupt model have sufficient accuracy for a consistent comparison with experimental data for $N_{s}$, which can be performed in, e.g., Shubnikov-de Haas and Hall experiments. Incidentally, the increase in $N_{s}$ as a consequence of the use of a non-abrupt doping profile predicted here shows that the theoretical underestimation of this quantity obtained with models based upon abrupt profiles should not be attributed solely to the presence of DX centers, as suggested by Ref. [25].

It must be emphasized that our nonuniform mesh choice is also important for obtaining a better simulation of the charge transfer process, since it has allowed us to allocate more points into the depletion region.

We also noted that the wave function penetration into the doped AlGaN barrier increases when a non-abrupt doping profile is adopted. This can be attributed to an increase in the number of ionized donors in the proximities of the 2DEG after the charge transfer process. Since this clearly increases the electron-ionized remote donors scattering rate, the electron mobility is reduced. Then our model makes way for more realistic simulations of electron transport in modulation-doped heterostructures.

For simplicity, we have not included residual acceptors in our self-consistent model. This is a point to be considered in future works, since it has been shown that the presence of background impurities can affect both the 2DEG density and the electron mobility in GaAs/AlGaAs one-side modulationdoped QWs [26]. To what extent this occurs in GaN/AlGaN one-side modulation-doped QWs is not well established in literature.

\section{CONCLUSIONS}

In this work, we present accurate self-consistent results for the dependence of the sheet density on the doping and interfacial profiles for electrons in a modulation-doped wurtzite GaN/AlGaN QW under the effects of the intrinsic polarization fields. A substantial increase in the 2DEG density is predicted for smaller spacer width and higher donor diffusion length. Other non-abrupt profiles for both interface and the doping are to be investigated. Since the effect on the 2DEG density is measurable, we expect availability of experimental results to fit our calculations.

Since the electrical characteristics of modulation-doped QWs greatly depend on the doping distribution [27], it is, in fact, important to include the impurity diffusion/segregation in the design of modulation-doped QWs-based electronic devices via the adoption of non-abrupt doping profiles.

\section{ACKNOWLEDGMENTS}

This work was partially supported by a grant from the Conselho Nacional de Desenvolvimento Científico e Tecnológico $(\mathrm{CNPq})$, and grants from CT-ENERG/CNPq N. 504.467/2004-2 and CT-PETRO/CNPq N. 550.126/2005-8. 
[1] Y. M. Park, Y. J. Park, K. M. Kim, J. C. Shin, E. K. Kim, M. H. Son, S. W. Hwang, and K. H. Yoo, Jpn. J. Appl. Phys. 41, 4375 (2002).

[2] E. C. Ferreira, J. A. P. da Costa, J. A. K. Freire, G. A. Farias, and V. N. Freire, Appl. Surf. Sci. 190, 191 (2002).

[3] V. V. Mitin, V. A. Kochelap, and M. A. Stroscio, Quantum Heterostructures: Microelectronics and Optoelectronics (Cambridge University Press, New York, 1999).

[4] E. Luna, A. Guzman, J. L. Sanchez-Rojas, J. M. G. Tijero, R. Hey, J. Hernando, and E. Munoz, J. Vac. Sci. Technol. B 21, 883 (2003).

[5] G. E. Kohnke, M. W. Koch, C. E. C. Wood, and G. W. Wicks, Appl. Phys. Lett. 66, 2786 (1995).

[6] Y. Huang, C. C. Williams, and J. Slinkman, Appl. Phys. Lett. 66, 344 (1995).

[7] P. A. Sundqvist, V. Narayan, S. Stafstrom, and M. Willander, Phys. Rev. B 67, 165330 (2003).

[8] J. S. Song, S. H. Seo, M. H. Oh, J. H. Chang, M. W. Cho, and T. Yao, J. Cryst. Growth 261, 159 (2004).

[9] C. Kisielowski, Z. Liliental-Weber, and S. Nakamura, Jpn. J. Appl. Phys. 36, 6932 (1997).

[10] T. Shirasawa, N. Mochida, A. Inoue, T. Honda, T. Sakaguchi, F. Koyama, and K. Iga, J. Cryst. Growth 190, 124 (1998).

[11] F. M. S. Lima, B. G. Enders, A. L. A. Fonseca, O. A. C. Nunes, V. N. Freire, J. A. K. Freire, G. A. Farias, and E. F. da Silva, Phys. Status Solidi C 1, S215 (2004).

[12] F. Bernardini, V. Fiorentini, and D. Vanderbilt, Phys. Rev. B 56, 10024 (1997).

[13] G. Bastard, Wave Mechanics Applied to Semiconductor Het- erostructures (Halsted, New York, 1988).

[14] T. L. Li and K. J. Kuhn, J. Comput. Phys. 110, 292 (1994).

[15] L. Bouzaiene, L. Sfaxi, H. Sghaeir, and H. Maaref, J. Appl. Phys. 85, 8223 (1999).

[16] G. Martin, A. Botchkarev, A. Rockett, and H. Morkoç, Appl. Phys. Lett. 68, 2541 (1996).

[17] N. Maeda, T. Nishida, N. Kobayashi, and M. Tomizawa, Appl. Phys. Lett. 73, 1856 (1998).

[18] O. Ambacher, J. Majewski, C. Miskys, A. Link, M. Hermann, M. Eickhoff, M. Stutzmann, F. Bernardini, V. Fiorentini, V. Tilak, et al., J. Phys.: Condens. Matter 14, 3399 (2002).

[19] V. Fiorentini, F. Bernardini, F. Della Sala, A. Di Carlo, and P. Lugli, Phys. Rev. B 60, 8849 (1999).

[20] A. F. Wright, J. Appl. Phys. 82, 2833 (1997).

[21] I. Vurgaftman and J. R. Meyer, J. Appl. Phys. 94, 3675 (2003).

[22] A. Zoroddu, F. Bernardini, P. Ruggerone, and V. Fiorentini, Phys. Rev. B 64, 045208 (2001).

[23] R. J. Krantz and W. L. Bloss, IEEE Trans. Electron Devices 36, 2593 (1989)

[24] Q. Fanyao, A. L. A. Fonseca, and O. A. C. Nunes, Superlattices Microstruct. 26, 377 (1999).

[25] A. M. C. Serra and H. A. Santos, J. Appl. Phys. 70, 2734 (1991).

[26] F. M. S. Lima, A. L. A. Fonseca, O. A. C. Nunes, F. Y. Qu, V. N. Freire, and E. F. da Silva, Physica E 17, 322 (2003).

[27] A. Leuther, A. Förster, H. Lüth, H. Holzbrecher, and U. Breuer, Semicond. Sci. Technol. 11, 766 (1996). 